\title{
Keck Observations of Candidate Ultra-Luminous X-ray Sources
}

\author{
Diane Sonya Wong, Ryan Chornock \\ and Alexei V. Filippenko
}

Astronomy Department, 601 Campbell Hall, University of California, Berkeley, CA 94720 USA

email: dianew@astro.berkeley.edu

\begin{abstract}
We present results of optical follow-up observations of candidate ultra-luminous Xray sources (ULXs). Using Keck optical spectroscopy, 17 of the candidates from the Colbert \& Ptak (2002) catalog have been identified; this is one of the largest sets of optical identifications of such objects thus far. 15 are background active galactic nuclei (AGN); 2 are foreground stars in our Galaxy. These findings are compared with background and foreground object expectations, as derived from $\log N-\log S$ relations. Also, the results are briefly discussed in terms of the spiral-galaxy/ULX connection.
\end{abstract}

Keywords. black hole physics, catalogs, galaxies: distances and redshifts, galaxies: individual (NGC 720, NGC 1316,NGC 1365, NGC 1399, NGC 2775, NGC 3266, IC 2597, NGC 3923, NGC 4151, NGC 4373, NGC 5128), quasars: general, X-rays: galaxies.

\section{Observations}

Ultra-luminous X-ray sources (ULXs; also referred to as intermediate-luminosity Xray objects [IXOs]) are non-nuclear point X-ray sources with apparent (isotropic) X-ray luminosities $L_{\mathrm{X}} \approx 10^{39}-10^{41} \mathrm{erg} \mathrm{s}^{-1}$. Colbert \& Ptak (2002, hereafter CP02) assembled the first complete catalog of candidate ULXs consisting of 87 objects listed in order of increasing right ascension.

Seventeen candidates (in 11 different galaxies) from the CP02 catalog were observed with the Keck 1 10-meter telescope on Mauna Kea, Hawaii, USA on 2003 February 28 and 2003 December 20-21 UT. Spectra were obtained using the Low Resolution Imaging Spectrometer (LRIS). The chosen setup yielded a wavelength coverage of $\sim 3150-9400 \AA$. A $1^{\prime \prime}$ wide slit was used all three nights. Typical seeing on these nights was $\sim 1^{\prime \prime}$. The resulting resolution was $\sim 6 \AA$.

Standard reduction was done with IRAF version 2.12 (flatfielding with dome flats, optimal extraction of the spectra, wavelength calibration) and IDL versions $5.5 \& 6.1$ (flux calibration, atmospheric band removal).

\section{Discussion}

We have found 15 of the candidate ULXs to be background objects with redshifts ranging from 0.239 to 2.784. The redshifts were found by fitting Gaussians to the line profiles, and comparison to a composite quasar spectrum. Additionally, 2 candidate ULXs are foreground objects. Of these, the counterpart of IXO 75 is actually a double M star. The results are shown in Figure 1.

The number of candidate ULXs reported by CP02 is the number found in a circle of radius $\mathrm{D} 25 \dagger$ for each galaxy. Using the Hasinger et al. (1998) $\log N$-log $S$ relation, the

$\dagger$ D25 is the galaxy's size as defined by the apparent isophotal diameter measured at, or reduced to, the surface brightness level of $25.0 \mathrm{~B}$ mag $\operatorname{arcsec}^{-2}$. 


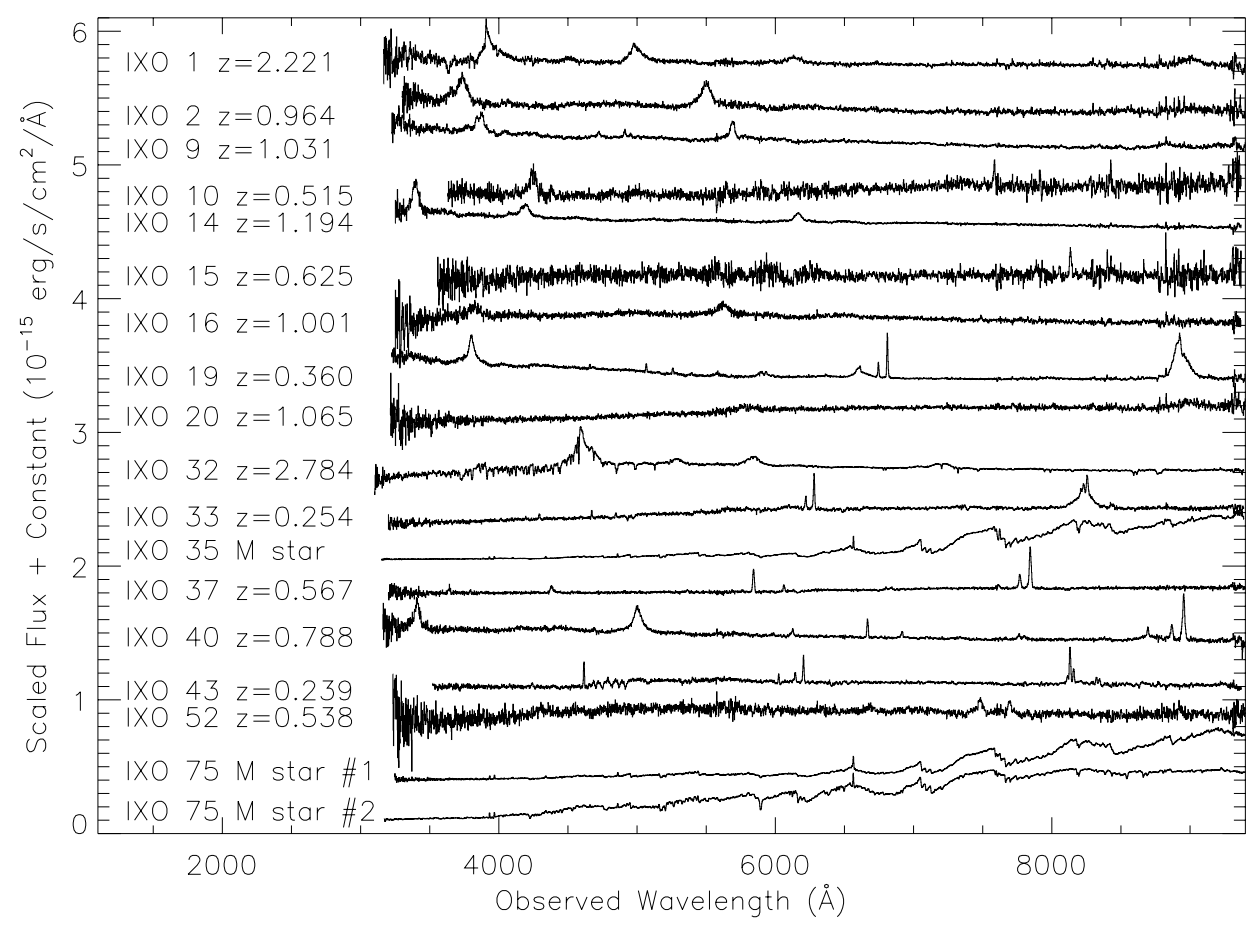

Figure 1. Candidate ULX Spectra. CP02 notation (e.g. "IXO 1") is used here. Most are AGN.

number of expected background and foreground sources ("fake" ULXs) was calculated for the same region. These two numbers were compared: the number of candidate ULXs reported by CP02 (24) is comparable to the expected number of "fake" ULXs (21) in these galaxies. Furthermore, the majority of the candidate ULXs we identified are outside of the D25 ellipse, so it is not surprising that they are not associated with the galaxies in whose fields they lie.

Of the remaining 70 candidate ULXs in the CP02 catalog, the majority (47) is in spiral galaxies. However, the mean of 1.3 candidates per spiral is comparable to (and slightly lower than) the mean of 2.3 per elliptical.

See the work by Gutiérrez \& López-Corredoira (2005) for another set of candidate ULX optical identifications.

\section{Acknowledgements}

The authors acknowledge NSF grant AST-0307894.

\section{References}

Colbert, E. J. M. \& Ptak, A. F. 2002, ApJS, 143, 25

Gutiérrez, C. M. \& López-Corredoira, M. 2005, ApJL, 622, L89, and these proceedings

Hasinger, G., Burg, R., Giacconi, R., Schmidt, M., Trumper, J. \& Zamorani, G. 1998, A\&̊A, 329,482 